\title{
Zum Problem der Bürgerbestimmung in der aristotelischen Politik
}

\author{
JAKUB JINEK \\ Charles University in Prague \\ jakub_jinek@hotmail.com
}

\section{Zusammenfassung}

Der Artikel wendet sich gegen die übliche Deutung der These von Aristoteles, der Bürgerbegriff richtet sich immer nach der Polis-Verfassung. Aristoteles proklamiert mit dieser Äußerung keinen bloß deskriptiven Gesetzpositivismus oder Verfassungsrelativismus. Er möchte damit eher seine Vorstellung des guten Polis-Lebens in den Vordergrund rücken, das aus einer Varietät der Lebensorientierungen besteht und das - insofern es allgemein wünschenswert ist - für alle Poleis normativ ist. Nur unter solchen Umständen kann die Verfassung als „das Eidos der Polis“ bezeichnet werden, an dem sich der Polites-Begriff orientieren soll. Der Bürger wird dadurch relativ breit aufgefasst und die gesamte Argumentation gleicht somit einem Plädoyer für die Volksverfassung (Politeia). Auf der anderen Seite stellt die Bürger-Definition eine wahrhaft aristotelische begriffliche Einschränkung, die ermöglicht, die Tugend des guten Mannes gegenüber der gemäßigten Normativität der bürgerlichen Tugend abzustufen und dadurch die guten Verfassungen auch gegeneinander zu differenzieren.

\section{Schlüsselwörter}

Aristoteles Politik, Bürgerbestimmung, Politische Verfassung, Staatsbürgerschaft

\section{Title}

On the Problem of Determining Citizens in Aristotelian Politics

\begin{abstract}
The paper disproves the widely held interpretation of Aristotle's statement that the concept of the citizen varies with the constitution. I claim that it gives no evidence for any positivism or constitutional relativism. What Aristotle truly intends here is to put emphasis on his idea of the good life in a city that consists of a variety of
\end{abstract}


forms and ways. This variety is generally desirable and thus prescriptive. It is only under these conditions that the constitution is "the Form of the city" which determines the citizenship. The concept of the citizen is thus conceived as rather broad and the argument reveals to be a plea for the rule of majority (polity). But on the other hand, the definition of citizen enables to distinguish the virtue of the good man from that of the good citizen und thus to differentiate among the good constitutions.

Key Words

Citizenship, Constitution, Polity, Aristotelian Politics

Recebido: 25/06/2015 - Aceptado: 11/12/2015

\section{Einleitung}

Was ist an der Aristotelischen Politik das wesentlich Neue? Worin besteht ihr systematischer Ertrag gegenüber der politischen Philosophie Platons? Diese Fragen führen uns gemeinhin zu Aristoteles' Kritik des platonischen Projekts der besten Verfassung im II. Buch seiner Politik zu. Die Konzentrierung am II. Buch ist in dem Sinne berechtigt, dass Aristoteles hier diejenigen Entwürfe Platons aufs Korn nimmt, die einerseits dem damaligen Publikum als die wesentlichsten und zugleich provokativsten vorkommen müssten, nämlich die Abschaffung des Privatbesitzes, der Familie und die Anforderung an die möglichst große Einheit der Gemeinschaft ${ }^{1}$, und auf die andererseits auch Platon besonders ankam (wie es deren zusammenfassende Wiederholung im Timaios bestätigt) ${ }^{2}$. Dennoch ermöglicht die Knappheit der hauptsächlich doxographischen Abhandlung des II. Buches nicht, die Kritik breiter anzulegen, und es bleibt oft bei der bloßen Behauptung der alternativen

1 Die für die Politeia zentrale Anforderung an die Philosophenherrschaft erwähnt Aristoteles dagegen nicht.

2 Platon, Tim. 17c-19a; Leg. V, 739a-e.

П Н Г Н / F O N S I (2016), 123-144 
Grundansichten ${ }^{3}$. Dieser Umstand macht die Kritik im II. Buch relativ wenig aufschlussreich für die Erkenntnis der eigentlichen Intention der Politik - natürlich wenn wir uns nicht damit zufrieden geben möchten, dass wir im II. Buch eine Kritik des totalitären Denkens sehen, um nur eines der verbreiteten Klischees zu nennen.

Viel wirksamer und im Bezug auf eine eigene Politikauffassung viel konstruktiver wird jedoch Platon dort angegriffen, wo Aristoteles im bewussten Opposition zu seinem Lehrer eine zwar begrifflich von ihm abhängige, inhaltlich aber wesentlich abweichende Konzeption formuliert: im I. Buch legt er ein alternatives Herrschafts- und Politikkonzept vor, in den Büchern IV. - VI. eine alternative Auffassung der wissenschaftlichen Gesetzgebung, in VII. - VIII. ein alternatives Model des besten Staates und Erziehungsprogramms. Eine besondere Stellung gebührt dennoch dem III. Buch der Politik. Dortige Abhandlung stellt eine direkte obwohl unausgesprochene - Auseinandersetzung mit dem methodologischen und systematischen Zentralpunkt der politischen Philosophie Platons dar, nämlich mit der Analogie von Polis und Seele. Der Angriff entfaltet sich zunächst unauffällig, um auf desto destruktivere Konsequenzen hinauszulaufen. Denn hier verwirklicht Aristoteles das, was er an der klassischen Stelle der Nikomachischen Ethik nur programmatisch behauptet, nämlich die methodische Absonderung der Politik von der Ethik ${ }^{4}$. Im vorliegenden Aufsatz möchte ich aufzeigen, wie Aristoteles im III. Buch der Politik diese Absonderung durchführt,

3 Wie auch immer diese Grundansichten in der Metaphysik beider Philosophen verankert sind (wie es z. B. Mráz 1998; oder ZEHNPFENNIG 2012 schildern). Vgl. KRAUT 2011, 52: „it is difficult to view these remarks as an effective rebuttal of Plato's proposal“. Erst die "soziologische“ These, die die Bedeutung der kleineren Gemeinschaften für die Existenz der Polis betont, die aber erst im 9. Kapitel des III. Buches formuliert wird, bietet - so Kraut (54-55) - eine effektive Widerlegung des platonischen Einheitsgedanken an. Vgl. auch KRAUT 2002, 306-307.

4 Aristoteles, Eth. Nic. X, 10,1179b1 ff. und 1181b14 ff.; vgl. $1177 \mathrm{~b} 26 \mathrm{ff}$.

П Н Г Н / F O N S I (2016), 123-144 
indem er einen a-moralischen, wohl aber sittlichen Begriff der Bürgerschaft entwickelt.

\section{Verschiedene Bürgerdefinitionen}

Dass Aristoteles im III. Buch seine eigene und ursprüngliche philosophische Agenda verfolgt, ist darin ersichtlich, wie schnell er die am Anfang der Erörterung angekündigte, in der zeitgenossischen Diskussion kanonische ${ }^{5}$ Frage nach dem Wesen und Qualität der Verfassung verlässt. Er macht somit klar, dass die Stadtverfassung keine allerwichtigste und selbständige Stellung in der politischen Theorie hat wie es bei Platon der Fall war -, sondern gänzlich von der Polis und deren Teilen - den Bürgern - abhängt ${ }^{6}$.

Und es ist gerade die enge Beziehung zwischen der Polis und dem Polisbürger, die die ganze folgende Untersuchung bestimmt, und die platonische Analogie von Polis und Seele ersetzt. Uns interessiert besonders die Eigentümlichkeit dieser Beziehung, die weder biologischgenetisch wie im Pol. I, 2, noch technisch-ökonomisch wie in den Pol. I, 4-7, aber auch nicht verfassungsrechtlich aufgefasst wird ${ }^{7}$. Besonders die letzterwähnte Konnotation scheint zwar für Aristoteles im III. Buch angesichts der hier untersuchten Themen (Verfassung, politische Ämter, Bürgerschaftsstatus) gewichtig $\mathrm{zu}$ sein, aber auch die Verfassungsproblematik ist schließlich - wie wir noch sehen werden durch die aristotelische Abkehr vom Verfassungsrechtlichen ${ }^{8}$ zum spezifisch Politischen betroffen. Obwohl Aristoteles im Fortgang seiner Abhandlung verschiedene Polis-Ämter und Institutionen auch

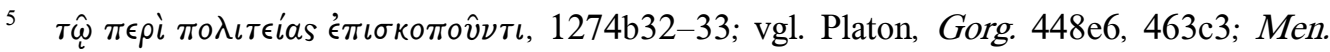
71b4, 86e, 87b3; vgl. Leg. VI, 767c1; Aristoteles, Rhet. I, 2, 1356a23, 6, 1362b5; Eth.

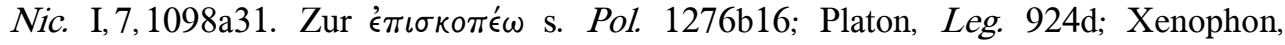
Mem. IV, 2, 24.

6 Vgl. SCHÜTRUMPF 1991, 382.

7 So Mulgan 1977, 66; und KeYT 1995, 130-132.

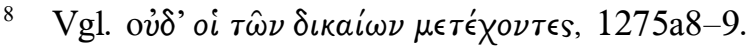


verfassungsrechtlich untersuchen muss, entwickelt er schließlich eine institutions- und verfassungsneutrale Konzeption der Bürgerschaft.

In den ersteren Kapiteln des III. Buches wird eine Reihe von Versuchsbestimmungen vorgelegt, die auf Definition des Bürgers schlechthin $(\dot{a} \pi \lambda \hat{\omega} s)$ zielen, d. h. auf eine solche, die keine zusätzliche Verbesserung brauchen würde ${ }^{9}$. Die erste Definition, die den Bürger durch Teilnahme am Gericht und Amt bestimmt ${ }^{10}$, zeigt sich als zu vag;

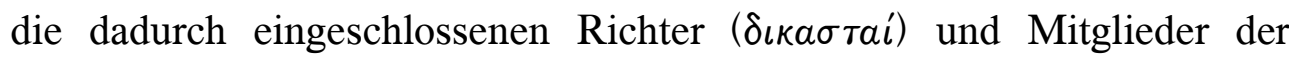

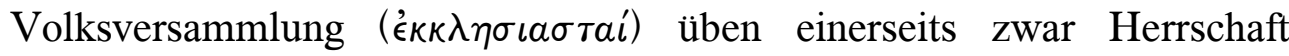

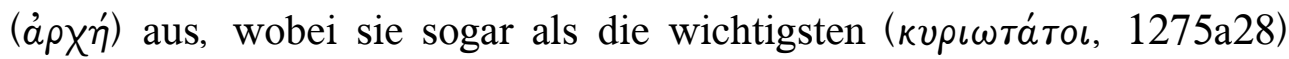
betrachtet werden müssen ${ }^{11}$, sie können aber anderseits zu den Herrschern

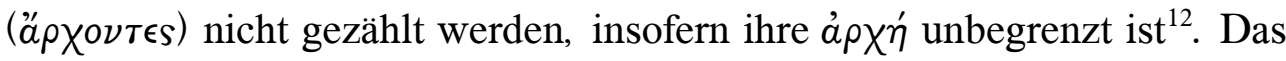
Problematische an der ersten Definition liegt also darin, dass sie schließlich keine wirkliche Bestimmung darstellt: Letztendlich kann man einem so los erfassten Bürger eine Regierungsrolle weder bedingungslos (s. $\dot{a} \pi \lambda \hat{\omega} s)$ zusprechen noch aberkennen.

Wenn man aber dieser Unbestimmtheit dadurch zu entgehen möchte, dass man - nun schon im Rahmen der zweiten Definition des Bürgers -

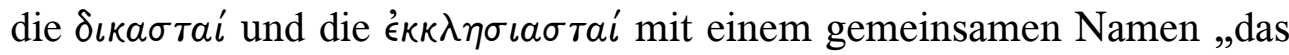

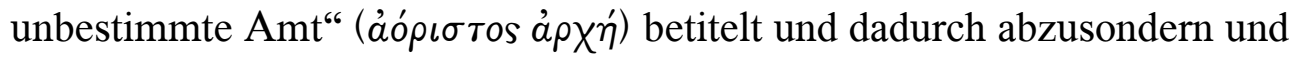

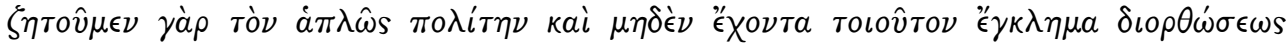
$\delta \epsilon o ́ \mu \epsilon \nu o \nu, 1275 \mathrm{a} 19-20$; vgl. a16, 22. Von dieser Bemerkung aus lässt sich die etwas schwer nachvollziehbare Methode und die sich daraus ergebende Vielheit der BürgerDefinitionen zu verstehen.

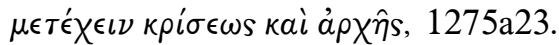

11 Dasselbe Wort stand am Anfang von Pol. I, 1 als Bezeichnug der Polis, die sich dadurch

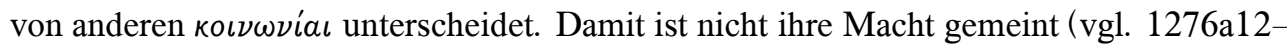
13), sondern eher ihre Würde, die darin besteht, dass die Polis die anderen

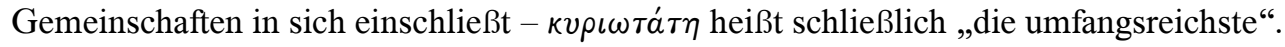
Aristoteles, Pol. 1275a27-29. 
zu begrenzen versucht ${ }^{13}$, taucht ein gleichsam umgekehrtes Problem auf. Eine solche Definition wäre zu eng und lediglich für demokratische Polisverfassungen passend ${ }^{14}$. Doch verbirgt diese zweite Definition eine Möglichkeit für Korrektion in sich ${ }^{15}$, was verhilft, die dritte Bestimmung zu formulieren. Der Polisbürger wird als derjenige ergriffen, wem die Erlaubnis oder Freiheit gegeben ist, an einem beratenden oder einem rechtsprechenden Amt teilzunehmen ${ }^{16}$. Diese Definition kann als endgültig gelten ${ }^{17}$, weil sie im Verlauf der weiteren Diskussion nie in Frage gestellt wird und weil sie zugleich - wie noch gezeigt werden soll Aristoteles' Zwecken sehr gut dient.

Der Unterschied zwischen dieser verbindlichen und beider vorangehenden unvollständigen Definitionen lässt sich mit dem Blick auf die Realität der athenischen Institutionen und Ämter beleuchten ${ }^{18}$. Das entscheidende Gedankenmanöver ist die Ersetzung der Angehörigkeit zur

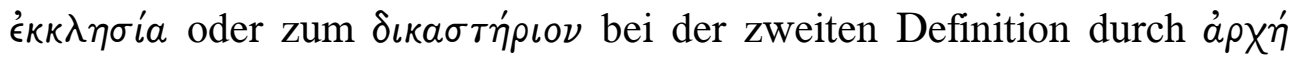

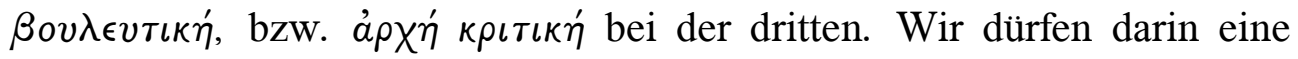
doppelte Absicht vermuten: a) Da der demokratische Rat ( $\beta$ ov $\left.\lambda \eta^{\prime}\right)$ ein

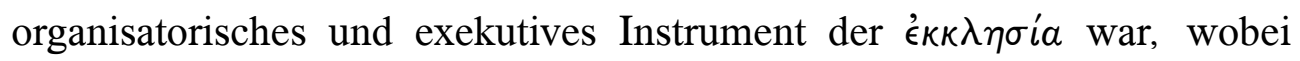
seine Mitgliedschaft restriktiv festgelegt wurde, kann auch dem Mitglied

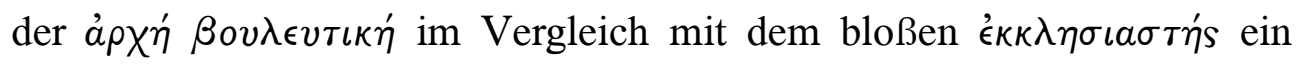
$\epsilon \sigma \tau \omega \delta \eta$
$1275 \mathrm{a} 31-33$.

Aristoteles, Pol. 1275a5.

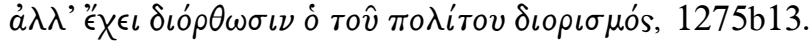

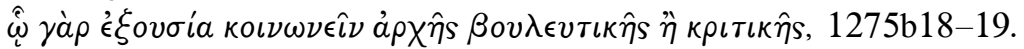

So auch BRAUN 1965, 21. Die weiteren drei Bestimmungsversuche (im 4., 5. und 13. Kapitel) können dann nur als Erweiterungen dieser dritten angesehen werden.

Dass hier Aristoteles eine demokratische Verfassungspraxis im Sinne hat, wird von seiner selbstkritischen Bemerkung erkennbar (1275b5). Diese reflexive Besinnung gibt uns aber andererseits zu verstehen, dass Aristoteles' Definition auf keine konkrete verfassungsrechtliche Ordnung zielt, sondern eher im Gegenteil die Ausschaltung der Probleme im Auge hat, die mit den empirischen Institutionsformen verbunden sind. Vgl. FREDE 2001, 77. 
engerer, und somit klar begrenzter Begriff der Bürgerschaft zugeschrieben werden. Auch die neuere Bestimmung des Bürgers durch die á $\rho \chi \eta^{\prime}$

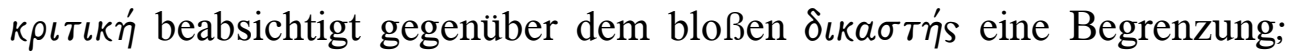
denn während zum Geschworenen eigentlich jeder Bürger gewählt werden konnte ${ }^{19}$, deutet die neuere Formulierung eher auf eine Befugnis, die den verschiedenen, mit bestimmter Agenda und Mitgliedschaft verbundenen Ämtern zukommt ${ }^{20}$.

b) Auf der anderen Seite möchte Aristoteles die diskutierten Befugnisse und Funktionen nicht einem konkreten, im institutionellen Rahmen der Demokratie oder Oligarchie verankerten Amt (etwa

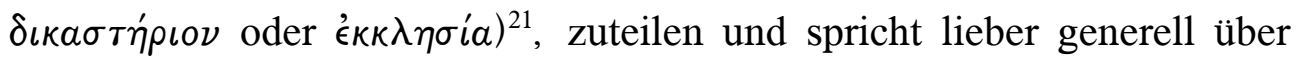
Ämtertypen, die er durch Verwendung eines Adjektivs abzugrenzen sucht

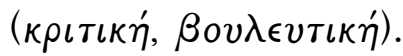

Das in der dritten Definition benutzte Wort $\mathfrak{\epsilon}^{\prime} \xi o v \sigma i a$ (Befugnis, Freiheit, Möglichkeit) hat im politischen Kontext zwei spezielle Bedeutungen, die wir im oben angeführten Zitat durch zwei Ausdrücken übersetz haben (Erlaubnis oder Freiheit). Einerseits drückt $\dot{\epsilon}^{\xi} \xi o v \sigma i ́ a$ eine Befugnis oder Erlaubnis aus, die einer Person von außen, durch Gesetz oder von Menschen, gebilligt wird ${ }^{22}$. Anderseits steht es für innere Möglichkeit, etwas zu tun, oder für Freiheit, sich beliebig, bloß aufgrund der eigenen inneren Entscheidung $\mathrm{zu}$ verhalten. In den meisten

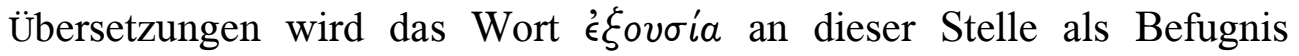

Vgl. FRITZ-KAPP 1977, 132.

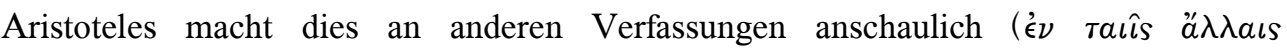

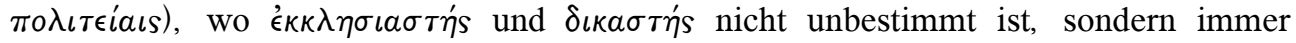

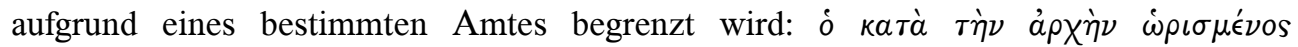
(1275b13-15).

21 Wie es gerade an der in der vorangehenden Fußnote zitierten Stelle der Fall war.

Wie etwa in 1275a25: " $€ \xi \epsilon \sigma \tau \iota \nu \stackrel{a}{\rho} \rho \chi \epsilon \iota \nu$. 
gedeutet $^{23}$. Ein Blick auf weiteres Vorkommen dieses Wortes in der Politik lässt uns dennoch nachdenklich werden. Wenn wir diejenigen Stellen beiseite lassen, die das gleiche Problem wie die jetzige Passage behandeln $(1255 b 35,1291 b 41,1315 a 14)$, und somit dem selben Deutungs-Dilemma ausgesetzt sind, ermöglichen die üblichen Stellen teils beide Auslegungen, wobei es aber immer um die Beziehung gerade zwischen dem menschlichen Willkür und dem Gesetz handelt (1270a21, 1286a13, 1302b9), und teils überwiegt eindeutig die spätere Möglichkeit,

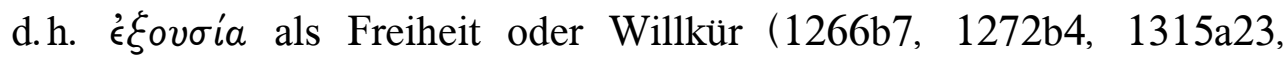
1318b39). Das altgriechische Politikverständnis hat offensichtlich besseren Sinn für die freie Spontaneität ${ }^{24}$ als die moderne, moralisierende Politik, die dazu neigt, Erlaubnis immer negativ auf dem Hintergrund eines Gebots zu verstehen: Erlaubt ist gerade das, was nicht verboten ist. Wenn wir an dieser Stelle die Übersetzung „Befugnis“ begünstigen, entkommt uns die eigentliche Absicht von Aristoteles und auch gewisse Kühnheit, mit der er diesen demokratischen Ausdruck in seine Konzeption einschlie $\beta \mathrm{t}^{25}$.

Das Wort $\epsilon^{\prime} \xi o v \sigma i ́ a$ schließt jedoch auch eine Begrenzung in sich ein, obschon eine viel subtilere ist als diejenige, die durch konkrete Gesetze und Normen erzwungen werden kann. Diese Begrenzung lässt sich

RACKHAM 1944: „who has the right“; ROLFES 1958: „wem es zusteht“; SCHWARZ 1989: „wem die Erlaubnis gegeben ist“; SCHÜTRUMPF 1991: „wem das Recht eingeräumt ist".

Vgl. Platon, Symp. 182e; Gorg. 461e, 526a; Aristoteles, Eth. Nic. 1114a16, 1163b19,

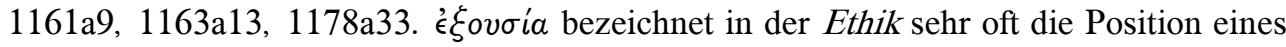
Herrschers: 1095b21, 1158a28, 1159a19. Die Bedeutung des Wortes ist also durchaus positiv, und entzieht sich der Negativität der modernen Vorstellung einer Befugnis.

Dadurch entsteht aber erst auch die moralische Tragweite dieser Freiheits-Vorstellung.

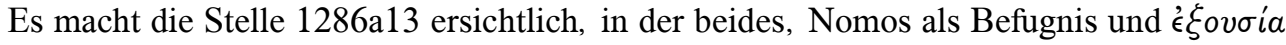
als Willkür nebeneinander gestellt werden. Man steht vor der Wahl: entweder kann man sich aufs Gesetz verlassen, und ausschließlich gesetzmäßig handeln, was ihm die Sicherheit bringt, nicht angeklagt zu werden, oder man wagt gesetzwidrig zu handeln, dann natürlich aber nur auf eigene Verantwortung. 
erblicken, wenn wir die Frage stellen, was es eigentlich heißen soll, dass jemand „Freiheit" hat, ein Amt zu bekleiden. Dies muss einerseits

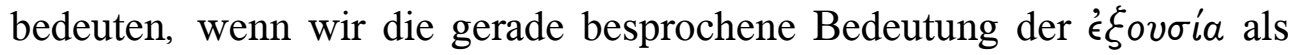
Spontaneität ernst nehmen sollen, dass die bürgerliche Freiheit auch einräumt, ein Amt nicht zu bekleiden. In anderen Worten, es liegt nur auf dem Bürger, ob er in den Rat kommt und seine Kontrollfunktion ausübt. Anderseits kann es doch - falls die dritte Definition der Bürgerschaft der Vagheit der ersten Versuchsbestimmung ausweichen soll - nicht nur Willkür und Beliebigkeit sein, die von der politischen (Nicht-)Beteiligung entscheidet. Im 5. Kapitel begegnen wir einen Kandidaten für eine subtile Begrenzung der '́\}̧一ovía des Bürgers, ein Amt innezuhaben. Die politische Teilnahme kann durch die Mangel an Zeit verhindert werden ${ }^{26}$. Der Bürger kann sich an den Ämtern beteiligen, wenn es ihm seinerseits möglich ist. Wer für Politik keine Muße hat, wird sich einfach nicht beteilen, obwohl seine Freiheit dazu formal immer besteht. Kein Gesetz

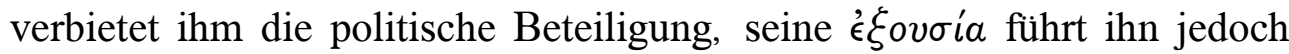
dazu, dass er selber erwägen muss, ob es ihm seine Lage erlaubt. Wenn Aristoteles sagt, eine gute Polis wird Arbeiter nicht zum Bürger machen (1278a8), muss er dabei nicht notwenig eine gesetzliche Restriktion im Sinne haben, sondern vielleicht eher die Beendung der problematischen Auszahlung des Lohns für die armen Mitglieder des Rats, die im 4. Jahrhundert notwendig wurde, um die demokratische Verfassung überhaupt zu erhalten (IV, 6, 1293a5) ${ }^{27}$. Das Problem der Besetzung der Ämter war aber - genauso wie heute - höchst delikat: einerseits war es notwendig, den Druck der verschiedenen parteiisch-ökonomischen Ansprüche standzuhalten, anderseits war aber immer schwieriger, die 
tatsächliche Teilnahme der Bürger zu bewahren ${ }^{28}$. Der Begriff $\mathfrak{\epsilon}^{\prime} \xi o v \sigma i ́ a$ ermöglicht Aristoteles, diese Spannung in seiner Konzeption zu berücksichtigen, ohne dabei eine oder andere Verfassungsordnung zu bevorzugen. Diese Lösung entspricht darüber hinaus völlig Aristoteles' Überzeugung von der zentralen Bedeutung der Muße für die erhabensten Tätigkeiten ${ }^{29}$.

Im Ganzen beobachten wir also in Aristoteles' Bürgerauffassung zwei einander entgegengesetzten Intentionen. Einerseits geht es ihm um die Bestimmung - ö $\iota \sigma \mu o s ~-$, die gegen demokratische - und in der athenischen Alltagspolitik wohl stark präsente - Unbegrenztheit und institutionelle Vagheit gestellt werden kann. Anderseits liegt ihm daran, die Bürgerbestimmung nicht auf konkrete bürgerisch restriktive Verfassung, besonders auf Oligarchie, oder aber auch auf Aristokratie, hinauslaufen zu lassen. Aristoteles möchte den Bürger $\dot{a} \pi \lambda \hat{\omega} s$, d. h. verfassungsrechtlich neutral erfassen.

Um diese spannungsvolle Bestimmung des Bürgers richtig zu verstehen, soll man nun zwei Fragen stellen: 1) Ist sie tatsächlich verfassungsneutral, auch wenn Aristoteles wiederholt auf die Verfassungsproblematik hinweist? 2) Was ergibt sich aus dieser Bestimmung für die Polis, d.h. schließlich für das ganze politische Projekt?

\section{Der Bürger und die Polisverfassung}

Die Verfassungsproblematik wird in die Diskussion im 2. Kapitel im Hinblick auf zeitgemäße politische Praxis einbezogen ${ }^{30}$. Aristoteles hat zwei Situationen im Sinne, einerseits die Zuteilung der Bürgerrechte

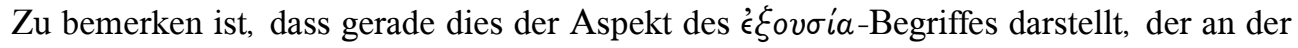
wichtigen Stelle Platonischer Nomoi im Zusammenhang des damals aktuellen Problems der Besetzung der Ämter besprochen wird (VI, 768b2). Aristoteles, Met. I, 1,981b13-25, I, 2, 982b20-24.

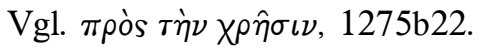


durch die Herkunft, anderseits die konfliktsvolle Lage bei der Abänderung der Polisverfassung. Im ersten Fall erwähnt er die ,politische und

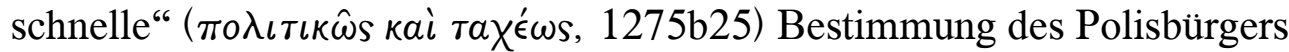
aufgrund der Herkunft seiner Eltern (wie sie etwa in athenischer Praxis

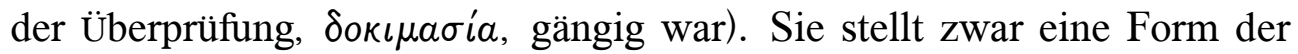
legalen Restriktion des Bürgerbegriffes dar, eine solche jedoch, die wohl nicht für die wissenschaftlichen Zwecke nutzbar sein kann, weil sie zum infiniten Regress in Form des „dritten Menschen“ führt: Die allerersten Bürger-Eltern wären gar nicht zu bestimmen (1275b32-34). Im zweiten Fall versucht Aristoteles das Dilemma, das aufgrund der unrechtmäßigen Verbreitung der Bürgerrechte in Folge der Verfassungsänderung entstand, durch die Unterscheidung zwischen dem Bürger, wie er tatsächlich ist, und dem Bürger zu Recht, zu lösen. Er lehnt dabei eine strikt normative Gleichsetzung beider Bürgerbegriffe ab, nach der nur der zu Recht gewordene Bürger als Bürger bezeichnet werden kann ${ }^{31}$. Diejenigen, die durch eine verfassungsrechtliche Abänderung zu den Bürgern gemacht wurden, haben diesen Status zwar nicht zu Recht, trotzdem aber insofern sie an einem Amt Anteil haben - Bürger genannt werden müssen ${ }^{32}$.

Dieses Resultat muss nun bekräftigt werden mit dem Hinblick auf die Identität der Polis ${ }^{33}$. Bei den Verfassungen, die das gemeinsame Nutzen

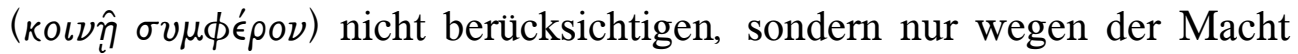
$(\kappa \rho a \tau \epsilon \hat{\imath} \nu)$ da sind, fällt Verfassung und Polis auseinander ${ }^{34}$. $\mathrm{Zu}$ diesen

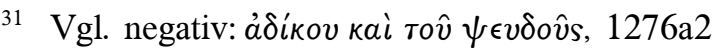

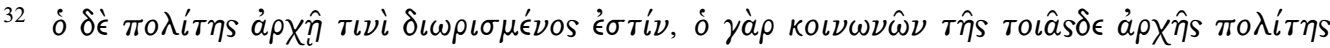

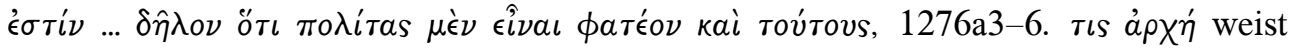
wahrscheinlich auf die vage zweite Bestimmung vom 1. Kapitel hin (vgl. $\dot{\omega} s \dddot{\epsilon} \phi a \mu \epsilon \nu$, a5). Vgl. auch åvaykaîov, 1278a16.

33 Dies war die Anfangsfrage der ganzen Untersuchung, auf die nun Aristoteles' Worte

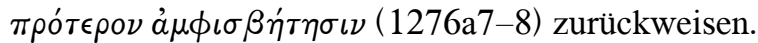

34 Ich bevorzuge hier die von Susemihl vorgeschlagene Lesung der Stelle 1276a14-16

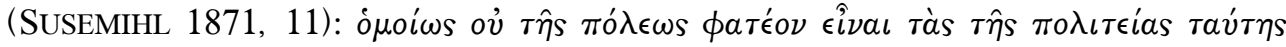


„Verfassungen ohne Polis ${ }^{635}$ gehört nicht nur Oligarchie und Tyrannis, sondern auch Demokratie, insofern sie Gemeinwohl ignoriert. Der Zusammenhang der Identitätsfrage und der Frage nach dem berechtigten Bürgersstatus $^{36}$ ermöglicht nun folgende Deutung: diejenigen, die nur aufgrund von konstitutionellen Veränderungen als Bürger bezeichnet werden (müssen), sind eigentlich keine Polis-Bürger, sondern nur Verfassungs-Bürger ${ }^{37}$.

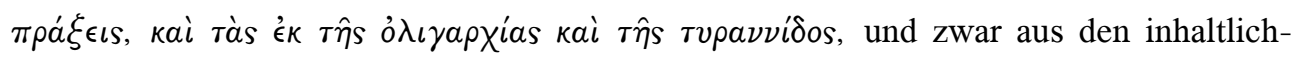
systematischen Gründen. Die entgegengesetzte Deutung (s. etwa NEWMAN 1902, 148), nach der die Täten der Polis und der beliebigen Verfassung zusammenfallen sollten, ist wenig sinnvoll: 1) Sie hätte die Frage nach der Identität der Polis vorzeitig entschieden. Wenn Aristoteles wirklich glaubte, Verfassung sei mit der Polis identisch, dann wäre es nicht nur eine gefährliche Annäherung an Platon gerade in dem Punkt, den Aristoteles in seiner Untersuchung in der Politik III zu umformulieren versucht, sondern es machte auch die ganze Untersuchung gar überflüssig. 2) Es stünde im direkten Widerspruch mit

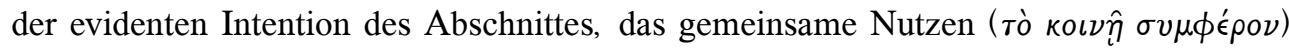

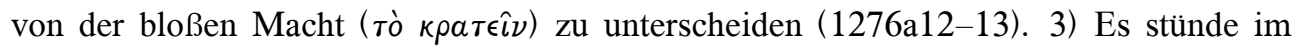
Widerspruch auch mit der gängigen Auffassung, auf der das Argument beruht, nämlich dass Tyrann sich von der Polis trennt, was einige zur Ablehnung der Verpflichtungen veranlasst. Wahrscheinlich wurde die Modifikation des Textes, die in den modernen Übersetzungen übernommen wurde, durch die Annahme verursacht, dass unsere Stelle die Behauptung am Ende des Kapitels antizipiert, nach der sich die Identität der Polis nach der Verfassung richte (1276b10-11). Diese Annahme beruht jedoch, wie noch gezeigt werden soll, auf einer unrichtigen Auslegung der letztgenannten Stelle. Vgl. SCHÜTRUMPF 1991, 403.

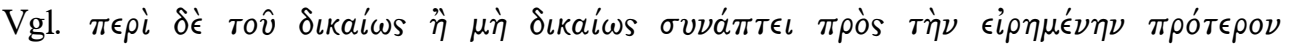

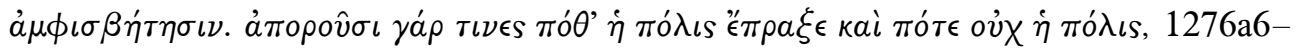
9. Das Bindewort $\gamma a ́ \rho$, das die Frage nach der Identität der Polis anführt, bezieht sich auf

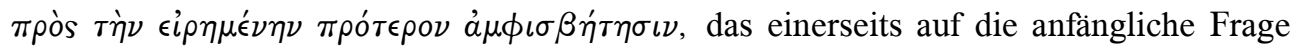
von 1274b34-36 und anderseits auf den unmittelbaren Kontext der Frage nach dem berechtigten Bürgersstatus hinweist.

Dieses Ergebnis steht im Einklang mit der wichtigen Stelle III, 7, 1279a31-32, der zufolge die Teilnehmer an der Polis entweder Anteil am gemeinsamen Nutzen haben müssen, oder dürfen sie nicht als Polisbürger genannt werden. 
Der Konzept der Bürgerschaft schlechthin ( $\dot{a} \pi \lambda \hat{\omega} s)$ oder zu Recht

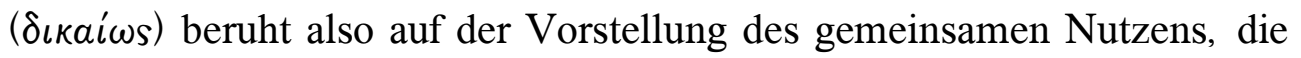
im III, 6 als Unterscheidungskriterium zwischen den richtigen, bzw.

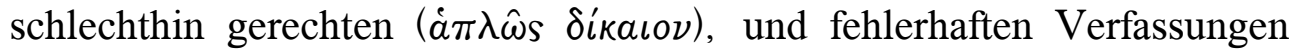
verwendet wird (1279a17-20). Das 6. Kapitel geht in diesem Punkt auf das I. Buch der Politik zurück mit dortiger Entgegensetzung der Sklavenherrschaft und der Freienherrschaft über Frau und Kinder (1255b18). Der Bürgerbegriff des III. Buches wird daher von der normativen Distinktionen im Haushalt, dem Bestandteil der Polis, wesentlich geprägt. Die für ihn relevante Spaltung ist folglich die zwischen dem gemeinsamen und eigenen Nutzen, d. h. zwischen der guten und schlechten Regierung.

Wenn Aristoteles am Ende des 3. Kapitels zum Ergebnis gelangt ist, man müsse bei der Identität der Polis die Verfassung berücksichtigen ${ }^{38}$, sollen wir es nicht als eine Feststellung über Abhängigkeit der Polis von der jeweiligen Verfassungsordnung verstehen, sondern eben als normative Bindung der Polis auf das Gemeinwohl, das in der Polisverfassung seinen besten Ausdruck findet. Dasselbe gilt folglich auch für die Identität des Bürgers zu Recht (bzw. schlechthin) und des Bürgers in der Tat - auch sie kommt nur in den Verfassungen zur Geltung, die schlechthin gerecht sind, d. h. deren Herrscher das Gemeinwohl verfolgen. Wenn kurz davor die Verfassung als $\epsilon \hat{i} \delta o s$ der Polis erfasst wurde ${ }^{39}$, und zwar im Zusammenhang, in dem die Polis als „Gemeinschaft von Bürgern der Staatsverfassung" definiert wurde ${ }^{40}$, ist dabei stark die Bindung der Verfassung auf $\kappa o \iota \nu \omega \nu \in \hat{\imath} \nu$, eine kooperative Lebensweise, und somit auch die normative Perspektive der bipolaren Gliederung auf gute und schlechte Regierungsformen, klar ersichtlich.

Aristoteles, Pol. 1276b2, 7.

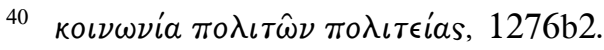

П Н Г Н / F O N S I (2016), 123-144 
Die andere Behauptung von Aristoteles, nämlich dass es aufgrund von Vielheit von Verfassungen auch eine Vielheit von Bürgersarten gibt ${ }^{41}$, muss mit dieser Deutung gar nicht in Konflikt geraten. Sie erklingt nämlich im Zusammenhang der Kritik der Demokratie, der schlechten Verfassung also, die das Bürgerrecht zu Unrecht auf viele verbreitet, so dass diese nur der Notwendigkeit halber als Bürger bezeichnet werden müssen. Aristoteles möchte in dieser Aussage über die Abhängigkeit des Bürgersstatus von der Regierungsform keinen positivistischen Verfassungsrelativismus billigen, von dem er von Anfang an als von einer fremden Ansicht berichtet $(1275 \mathrm{a} 2-5)^{42}$. Er möchte ihn eher philosophisch überwinden, und zwar aufgrund der Vorstellung von einer richtigen und unrichtigen Herrschaft.

Wir haben mit Aristoteles' Versuch zu tun, wissenschaftlich den Sachverhalt zu begreifen, dass einerseits ein normativer Begriff der Bürgerschaft besteht ( $\delta \iota k a i \omega s, \dot{a} \pi \lambda \hat{\omega} s$ ), dem anderseits nicht alle empirischen Erscheinungen entsprechen, die sich in das politische Leben

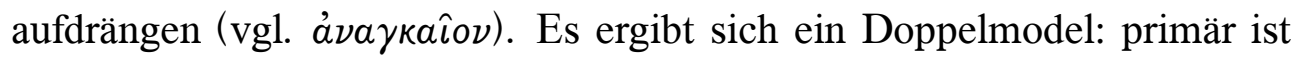
die Differenzierung der Verfassungen nach der Spaltungslinie gut und schlecht, die auf breiteren Kontext der Politik hinweist, wo dieses bipolare Model ihr Ursprung hat. Hier ist die Perspektive des Guten leitend, in deren Rahmen die Verfassung als $\epsilon \hat{i} \delta o s$ mit der Polis als einer gut organisierten und kooperierten Gemeinschaft wahrhaft zusammenfaillt. In der empirischen Perspektive, wo die schlechten Verfassungen in

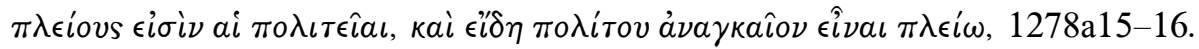

Aristoteles geht es also nicht um eine allgemeine Bürger-Definition, die überall angewendet werden kann, wie es Kahlenberg 1973, 105-106, deutet. Eine solche könnte dem Positivismus gar nicht widerstehen. Aristoteles geht es primär um die Definition des Bürgers, insofern er diesen Status zu Recht hat, und erst sekundär um die zahlreichen abweichenden Formen (1275b2-3). Im Zusammenhang mit der gerade zitierten Stelle ist es zu erwähnen, dass die Vielheit der Polisverfassungen für Aristoteles im III. Buch keinen bloß festgestellten Sachverhalt darstellt, sondern immer normativ als etwas Verhängnisvolles bewertet wird.

П Н Г Н / F O N S I (2016), 123-144 
näheren Betracht kommen, muss dann noch eine weitere Unterteilung auf

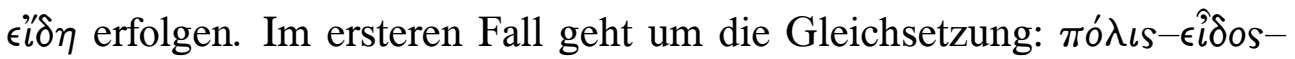
$\pi о \lambda \iota \tau \epsilon i a$, im späteren um die Unterscheidung vieler $\iota_{\epsilon} \iota \delta \eta$ der verschiedenen $\pi \circ \lambda \iota \tau \in i^{\prime} a \iota^{43}$.

Dieses Doppelmodell darf sich dabei auf Aristoteles' Metaphysik stützen. Denn dort finden wir auch zwei auf ersten Blick schwer auszusöhnende Auffassungen von $\epsilon \hat{\imath} \delta$ os. Einerseits ist es die Lehre von

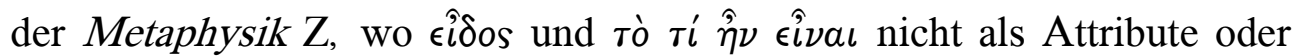
Prädikate einer jeglichen Sache dienen, sondern ausschließlich eigentliche Seins-Objekte, ovं íaı im primären Sinne sind, anderseits wird jedoch $\epsilon \hat{\delta} \delta$ os in der Kategorienschrift als die zweite ovं $\sigma$ ía erfasst, die das am wenigsten allgemeine, mehreren Subjekten zukommende Prädikat darstellt ${ }^{44}$.

\section{Bürgerliche Tugend}

Eine weitere Stelle, an der von der Abhängigkeit der Bürgerschaft von der Verfassung die Rede ist, ist im 4. Kapitel zu finden, wo Aristoteles zwischen der speziellen Tugend des Einzelnen und der gemeinsamen Tugend der Bürger unterscheidet. Die bürgerliche Tugend trägt dem Ziel des Ganzen zu, das in der Errettung der Gemeinschaft ( $\sigma \omega \tau \eta \rho i a)$ besteht, womit Aristoteles überraschend nicht die Rettung der Polis, sondern der

Wenn wir eine gute Polis beobachten, dann sehen wir eigentlich keine verfassungsrechtliche Pluralität, sondern schließlich nur ein $\epsilon \hat{\imath} \delta o s$, das die gute Polis wesentlich charakterisiert. Die dritte kanonische Bürgerdefinition bleibt somit ungetastet: der Bürger ist derjenige, dem es seinerseits möglich ist, an der Ausübung der amtlichen

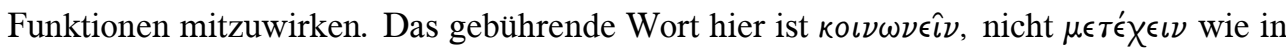
beiden ersteren Definitionen - es geht um das Gemeinsame, was nur durch Kooperation erzielt werden kann. Die Bürgerdefinition beruht nicht auf einem verfassungsrechtlichen Umstand, sondern auf einer fundamentalen Vorstellung der Polis als einer guten, kooperierenden Gemeinschaft.

44 Cat. 2a11-17; Met. Z,4,1029b13-14, 19-22, 1030a11-17. Vgl. SCHMITZ 1985, 5774. 
Polisverfassung meint. Da es eine Vielheit von Verfassungen gibt und da die Tugend der Bürger auf die Politeia hinblicken soll ${ }^{45}$, muss ein Unterschied zwischen der bürgerlichen Tugend und der Tugend des guten Mannes, die vollkommen und daher nur eine einzige ist, bestehen. Dem Argument liegt also der Gedanke der Abhängigkeit der Bürgerschaft von einer Vielheit der Verfassungen zugrunde. Es ist aber wichtig auf seinen Kontext aufzupassen. Die Rede ist nämlich von der besten Polis und der besten Verfassung (1277a77; 1276b37), und somit werden hier lediglich die richtigen Verfassungen differenziert. Diese Differenzierung erfolgt aufgrund des Unterschieds zwischen dem guten Bürger und dem guten Menschen.

Aristoteles möchte durch diese Unterscheidung einen selbständigen Begriff der bürgerlichen Tugend aufsuchen, ohne dabei aus der Polis eine Tugendgemeinschaft zu machen, so wie sie etwa in platonischer Politeia dargestellt wurde ${ }^{46}$. Sobald die gute Polis ohne den guten Menschen als der Gegenstand der Politikwissenschaft etabliert ist, wird die platonische Analogie von Polis und Seele schlussendlich aufgelöst.

Aus dem Unterschied zwischen dem guten Mann und dem guten Bürger ergibt sich, dass es möglich ist, ein rechtschaffener Bürger zu sein ohne den Besitz der Tugend, nach der ein rechtschaffener Mann bestimmt is $t^{47}$. Es besteht nach Aristoteles keine Notwendigkeit, vom guten Bürger auch innere ethische Beschaffenheit zu verlangen. Der gute Bürger ist von der moralischen Belastung, die auf ihn Platons Politeia aufgelegt hat, befreit. Das Wort für Konzession, é $\nu \delta \dot{\epsilon} \chi € \epsilon \tau a l$, gehört zum denselben Gedankenfeld wie $\dot{\epsilon} \xi o v \sigma i a$; sie konstituieren gemeinsam die Vorstellung der politischen Freiheit, die die ganze Sphäre von Politik gezeichnet und bestimmt. Das Politische wird nun eindeutig zum Nicht-Ethischen, mit

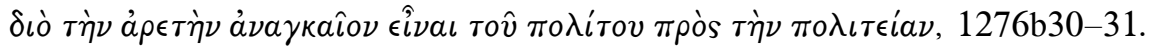

Oder so wie sie nach Aristoteles im Haushalt besteht (Pol. I, 13).

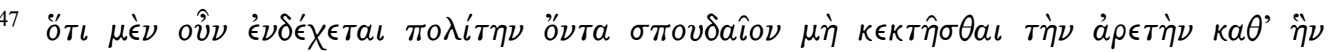

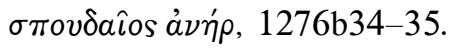


einer Entschiedenheit, die am Ende der Nikomachischen Ethik noch kaum $\mathrm{zu}$ ahnen war. Um aber nicht relativistisch $\mathrm{zu}$ argumentieren, muss zugleich eine eigens politische Normativität erarbeitet werden, eine Sittlichkeit, die in der Tugend des guten Bürger besteht.

Diese Tugend bringt den guten Bürger in die Lage, sowohl $\mathrm{zu}$ herrschen als auch beherrscht zu werden ${ }^{48}$. Bei dieser Bestimmung, die als vierte, (im Bezug auf die dritte) zusätzliche Definition des Bürgers angesehen werden kann, verlässt sich Aristoteles auf die Richtigkeit der allgemeinen Empfindungen ${ }^{49}$, die allerdings seiner Konzeption sehr günstig entgegen gehen, indem sie die in der dritten, verbindlichen Bürgerdefinition präsente innere Spannung zwischen Freiheit und Begrenzung erklären: Die Beschränkung der Teilnahme an der Regierung wird jetzt als Abwechselung gefasst. Diese Abwechselung kann als Gerechtigkeitsanforderung verstanden werden, weil sie die Belastung, die die Regierenden als Dienst zugunsten der anderen an sich nehmen, auf verschiedene Personen abwechselnd verteilt. Von der verkehrten Perspektive aus gesehen kann die Abwechselung vielen, die als Individuen keine Muße haben, die für die Politik förderliche Freizeit

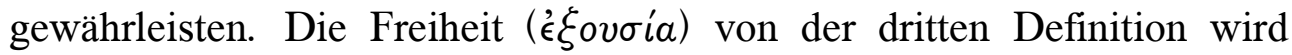
durch die Vorstellung der beiderseitigen Hilfe bereichert.

Der Gedanke der bürgerlichen Tugend lässt allerdings eine wichtige Ausnahme zu. Diese betrifft die Person des guten Mannes ${ }^{50}$, bei dem die bürgerliche und menschliche Tugend schlechthin einen einzigen innerlichen Zustand darstellt ${ }^{51}$. Seine einmalige und herrschende Tugend 


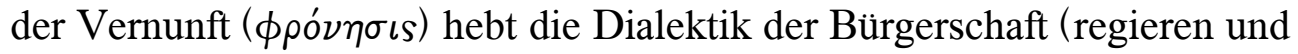
regiert werden) auf, indem sie beides auf eins bringt ${ }^{52}$. Der gute Mann -

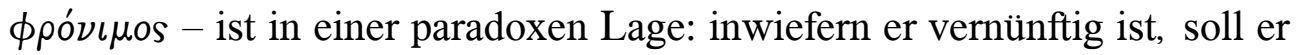
bei der Herrschaft nie abgewechselt werden; trotzdem aber gehört beides - d. h. regieren und regiert werden - auch zu ihm (1277b16-17). Aristoteles schildert das Verhältnis seiner Tugend $\mathrm{zu}$ üblichen bürgerlichen Tugenden in der Weise, die teils an die Beziehung zwischen dem Haushaltsherr und seinen Untertanen (Sklave, Frau, Kind) im Pol. I, 13 (1277b21-25), teils sogar auf die Beziehung zwischen den Polisschichten in Platons Politeia, erinnert ${ }^{53}$.

Es scheint also, dass Aristoteles diese Figur für die potentielle Differenzierung der guten Regierungsformen benutzt, ohne dabei die dritte und vierte verbindliche Definition des Bürgers verlassen zu müssen.

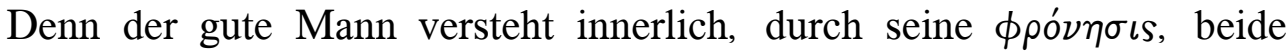
Seiten des Regierungsprozesses: Wenn in der guten Polis eine ausreichende Zahl der Bürger vorkommt, die der dritten Definition entsprechen, teilt er gerne seine Herrschaftsrolle mit ihnen. Er ist bereit, sich der Mehrheitsüberlegenheit zu unterwerfen, obwohl diese Willigkeit bei ihm nicht aus der äußeren Notwendigkeit ${ }^{54}$, sondern immer aus seiner inneren Freiheit und Einsicht hervorgeht. Wenn es aber den anderen Einwohnern nicht möglich ist, die amtlichen Aufgaben zu erfüllen, dann muss die Regierungsdialektik, die in der Bürgertugend erhalten ist, enger

Verschiedene Bürgertugenden haben dagegen eine unterschiedliche Beziehung zur Einheit: sie sind harmonisierend miteinander, und zwar im Bezug auf das Ziel, die

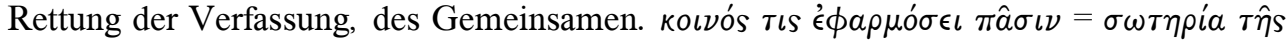

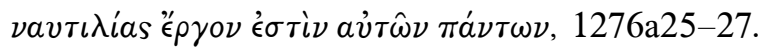

53

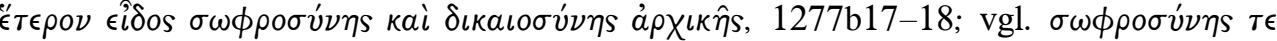

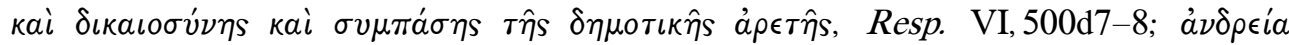

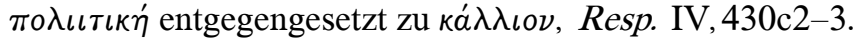

54 Oder aus seiner Angewohnheit, so wie bei den anderen, die die Herrschaftsrolle als beherrschten erlernen müssen; $1277 \mathrm{~b} 10$. 
ausgelegt werden, so dass sich eine teilnehmerisch restriktivere Verfassung daraus ergibt ${ }^{55}$.

Wenn wir nun zu jener Aussage von Aristoteles zurückkehren, der

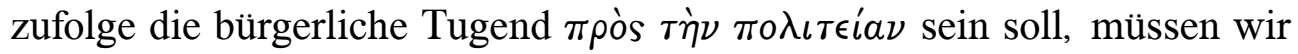
sie in einer Doppelperspektive des Zieles der besten Polis und der in ihr bestehenden sittlichen Kondition verstehen. Das Ziel ist das

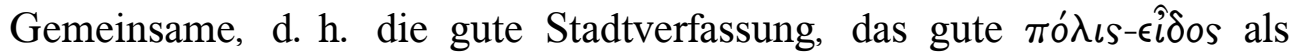
Ausdruck des besonderen guten Lebensstil, das durch rechtmäßige Abwechselung der Regierungsrollen zustande kommt und erhalten wird ${ }^{56}$. Die konkrete Art und Weise, wie dies erzielt werden kann, unterschiedet sich jedoch je nach der tatsächlichen Personal-Lage in der Polis. Beides, das Ziel und die Kondition, steht in keinem Widerspruch zueinander und es wird in dieselbe verbindliche verfassungsneutrale Definition der Bürgerschaft einbezogen.

\section{Schluss}

Das politische Denken von den Griechen wurde spätestens seit Herodotos durch die Intuition geprägt, der zufolge das politische Leben sich am treffendsten in der Frage der Verfassungsform widerspiegelt. Platon hat diese Intuition vertieft und mit der Seeleproblematik

Diese Möglichkeit scheint jedoch in der politischen Praxis nicht sehr wahrscheinlich zu sein und Aristoteles führt es wohl eher als eine hypothetische Möglichkeit vor (vgl. III, 13,1284a10). Der Zusammenfassung im 13. Kapitel zufolge gehört auch die Aristokratie zu den reinen Verfassungstypen, bei denen je nur eine Polisschicht und ihr Legitimationskriterium dominant ist. In einer tatsächlichen, geschichtlichen Polis, wo alle Gruppen und alle Kriterien zugleich da sind, dürfen sie sich diese nicht gegenseitig ausschließen und zum Nachteil der anderen überwiegen.

In diesem Sinne sagt Aristoteles im IV. Buch, die Verfassung sei „das Leben der Polis“.

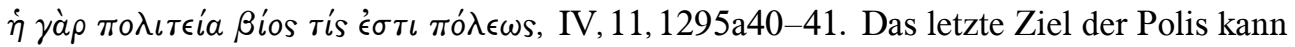
man nach III, 9 folgend verstehen: als die Gemeinschaft des guten Lebens sowohl für die Häuser und für die Geschlechter um eines vollendeten und selbstgenügsamen Lebens willen. $1280 \mathrm{~b} 32 \mathrm{ff}$. 
verbunden. Aristoteles wendet sich gegen diese traditionelle Agenda der politischen Wissenschaft und relativiert - wenn nicht $\lambda o ́ \gamma \omega$, dann aber sicher $\epsilon^{\prime} \rho \gamma \omega$ - die Frage der Verfassung als die für die Politik leitende. Er betont dagegen die Rolle der freien Bürgerschaft und des genuin politischen Zieles, das im guten Leben im Rahmen der Polis liegt.

Das sich daraus ergebende Auseinanderfallen der Analogie von Polis und Seele, Absonderung der Ethik von der Politik bedeutet allerdings keine Verlassung des normativen Anspruchs für die Politik. Der gute Bürger braucht zwar nicht mehr gute Seele zu haben, er muss aber die ihm gebührende Rolle vollfüllen, denn es geht immer um die beste Polis $^{57}$. Es ist gerade diese Perspektive, die - aufgrund der reflektierten methodischen Wechsel - in den neuen Bürgerschaftsbegriff auch die empirischen Nebenformen einzuschließen weiß.

Es ergibt sich ein Bürgerbegriff, der - in metaphysischen Termini

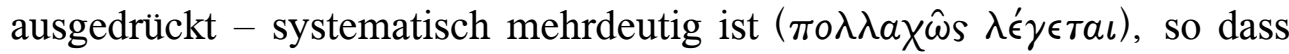

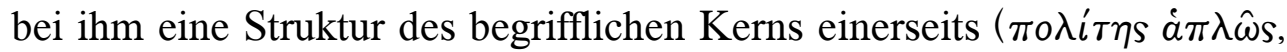

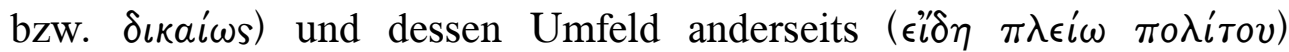
vorkommt. Das Umfeld, das aus verschiedenen $\epsilon i \delta \eta \eta$ besteht, bezieht sich zum Kern, der dem einzigen $\epsilon \hat{\imath} \delta o s$, analogisch. Die Beziehung zwischen dem bürgerlich und menschlich guten (oder schlechthin guten) Mann lässt sich metaphysisch als $\pi \rho \grave{s} \stackrel{\bullet}{\epsilon} \nu$-Verhältnis auszudrücken ${ }^{58}$. Durch $\pi \rho \grave{s} \stackrel{\text { }}{\epsilon} \nu$ kommt die typisch aristotelische Normativität zur Geltung, die nicht dem

Sie ist im doppelten Kontext die beste: a) sie ist vollkommen durch ihre innere Spannung zwischen der Begrenzung und Freiheit, Teilnahme und Entlassung (weswegen sie auch einen rechtlich neutralen Bürgerstatus verlangt), denn nur eine solche ermöglicht das potenzielle Engagement des besten Mannes, der nie zum Tyrann werden darf; b) sie schaut auf ihr einheitliches Ziel, das im guten gemeinsamen Leben besteht und zu dem auch verschiedene Personen unterschiedlich beitragen.

Die Bestimmung der bürgerlichen Tugend $\pi \rho \grave{s} \tau \grave{\eta} \nu \pi o \lambda \iota \tau \epsilon i a \nu$ bekommt dadurch eine überraschende Pointe. 
Gedanken der hierarchischen Ordnung entspringt, sondern teleologisch bei der Phänomenologie des politischen Lebens ansetzt ${ }^{59}$.

\section{Bibliographie}

Bleicken, J. (1986), Die athenische Demokratie, Padeborn, Schöning.

BRAun, E. (1965), Das dritte Buch der aristotelischen Politik. Interpretation, Wien, Böhlaus.

FREDE, D. 2011, „Staatsverfassung und Staatsbürger“, in: O. HöFFE (ed.), Aristoteles” Politik, Berlin, Akademie Verlag, 63-78.

FRITZ, K. VON-KAPP, E. (1977), „The Development of Aristotle's Political Philosophy and the Concept of Nature", in: J. BARNES, M. SCHOFIELD, R. SORABJI (eds. ), Articles on Aristotle, 2. Ethics and Politics, London, Duckworth.

KAHLEnBerg, K. (1973), „Zur Interpretation von Buch III der Politik“, in: P. SteInMetz (ed.), Schriften zu den Politika des Aristoteles, Hildsheim, Olms, 102179.

Keyt, D. (1995), „Supplementary Essay“, in: R. RoBInson (ed.), Aristotle. Politics, III-IV, Oxford, Claredon.

Kraut, R. (2002), Aristotle. Political Philosophy, Oxford, OUP.

KraUt, R. (2011), „Aristotle's Critique of False Utopias“, in: O. HöFFE (ed.), Aristoteles' Politik, Berlin, Akademie Verlag, 49-62.

MráZ, M. (1998), „Die Kritik an Platons Politeia im II. Buch von Aristoteles` Politik“, in A. HAVLíčEK-F. KARFíK (eds.), The Republic and the Laws of Plato, Prague, OIKOYMENH, 76-88.

Mulgan, R. (1977), Aristotle's Political Theory, Oxford, Clarendon.

Newman, W. L. (1887-1902), The Politics of Aristotle, I-II, Oxford, Clarendon.

RaCKham, H. (1944), Aristotle. Politics, London, Heinemann.

Rolfes, E. (1958), Aristoteles. Politik, Hamburg, Meiner.

Schmitz, H. (1985), Die Ideenlehre des Aristoteles. I: Kommentar zum 7. Buch der Metaphysik, Bonn, Bouvier.

SCHüTrumpF, E. (1991), Aristoteles. Politik, II-III, Berlin, Academie Verlag.

Schwarz, B. (1989), Aristoteles. Politik, Stuttgart, Reclam.

STOCKS, J. S. (1936), „Scholé“, «The Classical Quarterly» 30, 177-187.

Die Verfassung des Artikels wurde vom Projekt Nr. 15-13399S der Tschechischen Agentur für die Unterstützung der Wissenschaft unterstützt.

П Н Г Н / F O N S I (2016), 123-144 


\section{Jakub Jinek}

Suseminl, F. (1871), De Aristotelis Politicorum libris tribus prioribus quaestiones criticae, Greifswald, Kunike.

ZEHNPFENNIG, B. (2012), „Die aristotelische Platonkritik“, in B. ZEHNPFENNIG (ed. ), Die Politik des Aristoteles, Baden-Baden, Nomos, 37-55. 